\title{
Bilateral sequential lung transplantation in Kartagener syndrome
}

\author{
Kwok Fai Lucius Lee, MB ChB (CUHK), FRCSEd (CTh), \\ Kuan Yew Michael Hsin, MBBChir (Cantab), FRCSEd (CTh), \\ Ko Yung Alva Sit, MBBS (HK), FRCSEd (CTh), Ka Lai Cally Ho, MBBS (HK), FRCSEd (CTh), and \\ Wing Kuk Timmy Au, MBBS (NSW), FRCSEd (CTh), Hong Kong
}

\footnotetext{
From the Department of Cardiothoracic Surgery, Queen Mary Hospital, Hong Kong.

Disclosures: The authors reported no conflicts of interest.

The Journal policy requires editors and reviewers to disclose conflicts of interest and to decline handling or reviewing manuscripts for which they may have a conflict of interest. The editors and reviewers of this article have no conflicts of interest.

Received for publication June 11, 2020; revisions received June 11, 2020; accepted for publication June 17, 2020; available ahead of print June 24, 2020.

Address for reprints: Kwok Fai Lucius Lee, MB ChB (CUHK), FRCSEd (CTh), Department of Cardiothoracic Surgery, Queen Mary Hospital, Pok Fu Lam Rd, Room 308, New Clinical Building, Hong Kong (E-mail: luciusleekwokfai@gmail.com).

JTCVS Techniques 2020;3:406-8

2666-2507

Copyright (c) 2020 The Authors. Published by Elsevier Inc. on behalf of The American Association for Thoracic Surgery. This is an open access article under the CC BY-NC-ND license (http://creativecommons.org/licenses/bync-nd/4.0/).

https://doi.org/10.1016/j.xjtc.2020.06.025
}

Video clip is available online.

Kartagener syndrome (KS) is a rare congenital disease characterized by a triad of sinusitis, bronchiectasis, and situs inversus. Bronchiectasis can progress to end-stage lung failure, and lung transplantation (LTx) might be the only effective treatment. LTx in patients with KS has been technically challenging; however, available reported data are scarce. We have described 2 cases of bilateral sequential LTx in patients with $\mathrm{KS}$ and highlighted the pitfalls encountered. The institutional review board of the University of Hong Kong approved the study protocol, and the included patients provided written informed consent.

\section{CASE REPORT \\ Patient 1}

Our first patient was 40-year-old nonsmoking woman with KS and advanced bronchiectasis requiring repeated hospital admissions for infective exacerbation. She had been listed for LTx in 2013 and had undergone bilateral sequential LTx in 2015. The donor and recipient characteristics are listed in Table 1. During the recipient procedure, a clamshell incision was used, and chest entry was performed through the fourth intercostal space. We inserted a Medtronic EOPA 18F arterial cannula and Medtronic Carmeda

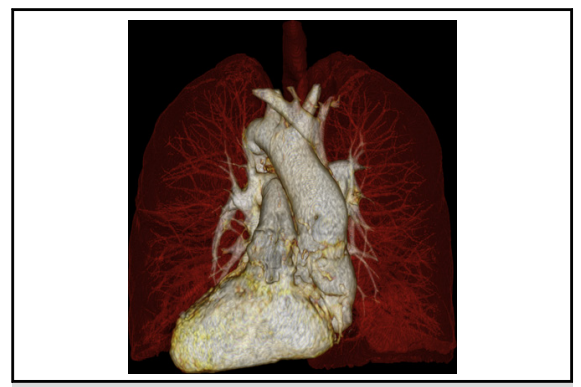

3D reconstruction of intrathoracic organs in a patient with Kartagener syndrome after bilateral sequential lung transplantation.

\begin{abstract}
CENTRAL MESSAGE
Implanting lungs from donors

with situs solitus to recipients

with Kartagener syndrome is

challenging. Understanding the

anatomic discrepancies and

careful preoperative planning are

necessary for a successful

operation.
\end{abstract}

See Commentaries on pages 409 and 410.

venous cannula (Medtronic, Dublin, Ireland) into the ascending aorta and right atrium, respectively, and venoarterial extracorporeal membrane oxygenation (ECMO) support using the Cardiohelp system (Maquet, Rastatt, Germany) was begun. The recipient's morphologically right (anatomically left) lung was explanted. The right main bronchus was anastomosed, followed by left atrial anastomosis. The donor pulmonary artery (PA) had to be transposed in a posterosuperior direction to anastomose it to the superiorly located recipient PA. On the left side, the donor PA was displaced anteriorly, circumventing the left main bronchus (Figure 1,A). The remainder of the left lung implant was performed in accordance with standard practice. The duration of ECMO support was 5 hours, $44 \mathrm{mi}-$ nutes. Because of the significant size mismatch, nonanatomic lung volume reduction over the right middle lobe 
TABLE 1. Donor and recipient characteristics

\begin{tabular}{|c|c|c|}
\hline Characteristic & Patient 1 & Patient 2 \\
\hline Height, cm & 160 & 162 \\
\hline Weight, kg & 37 & 54 \\
\hline $\mathrm{FEV}_{1}, \mathrm{~L}$ & $\begin{array}{c}0.53 \\
(19 \% \text { predicted })\end{array}$ & $\begin{array}{l}0.92(34 \% \\
\text { predicted })\end{array}$ \\
\hline pTLC, L & 4.4 & 5.0 \\
\hline $\mathrm{pO}_{2}, \mathrm{kPa}$ & 10.9 & 7.3 \\
\hline $\mathrm{pCO}_{2}, \mathrm{kPa}$ & 7.4 & 5.4 \\
\hline $6 \mathrm{MWT}, \mathrm{m}$ & $\begin{array}{c}318\left(\mathrm{SpO}_{2},\right. \\
88 \% \text { to } 82 \%)\end{array}$ & $\begin{array}{c}400\left(\mathrm{SpO}_{2}\right. \\
95 \% \text { to } 76 \%)\end{array}$ \\
\hline Pulmonary hypertension & No & mPAP $35 \mathrm{~mm} \mathrm{Hg}$ \\
\hline Donor age, y; gender & 39; Female & 61; Male \\
\hline Donor pTLC, L & 4.0 & 5.4 \\
\hline
\end{tabular}

$F E V_{l}$, Forced expiratory volume in 1 second; $p T L C$, predicted total lung capacity; $p O_{2}$, partial pressure of oxygen; $p C O_{2}$, partial pressure of carbon dioxide; $6 M W T$, 6-minute walk test; $\mathrm{SpO}_{2}$, peripheral oxygen saturation; $\mathrm{MPAP}$, mean pulmonary arterial pressure.

and left lingular segment was necessary. Postoperatively, she had experienced persistent air leakage and grade 1 acute rejection, which was confirmed by transbronchial biopsy in the first month. Otherwise, she had recovered uneventfully and was discharged on postoperative day 35 . At the 5-year follow-up examination, she remained well, ambulatory, and free of graft dysfunction or major infection.

\section{Patient 2}

Our second patient was a 51-year-old man with KS. His chest condition had deteriorated progressively, leading to a home oxygen therapy requirement since 2018. Bilateral sequential LTx was performed in 2019. The listing criteria and donor information are shown in Table 1. Intraoperatively, morphologically left (anatomically right) pneumonectomy was performed first. The same technical modifications with regard to bilateral PA anastomosis were required as described for patient 1 . The procedure was then repeated on the right side. Venoarterial-ECMO support (Cardiohelp system; Maquet) was established using a Medtronic Biomedicus 17F arterial cannula and Medtronic Biomedicus $23 \mathrm{~F}$ venous cannula via the right femoral vessels to achieve better exposure and allow retraction of the heart. The postimplantation anatomy of the airway and hilar structures is depicted in Figure 1, $B$. The total ECMO support time was 2 hours, 22 minutes. He experienced prolonged air leakage and was discharged on postoperative day 28. His post-LTx forced expiratory volume in 1 second was $3.1 \mathrm{~L}$, and his 6-minute walk test distance was $510 \mathrm{~m}$. At his 1-year follow-up examination, he had not experienced rejection, graft dysfunction, or infectious complications.

\section{DISCUSSION}

Situs inversus is pathognomonic in patients with KS. However, those who require LTx will almost invariably receive lungs from patients with normal situs. Because the left lung is not identical to the right lung, the anatomic discrepancies between the donor and recipient will make LTx technically demanding. Some investigators have proposed anastomosing the donor PA to the side of the recipient PA; however this carries the risk of stump stasis. ${ }^{1}$ Failed graft implantation was even reported in 1 patient with concomitant scoliosis. ${ }^{2}$ With adequate PA length and mobilization, we were able to perform an end-to-end anastomosis by swapping the donor PA between the
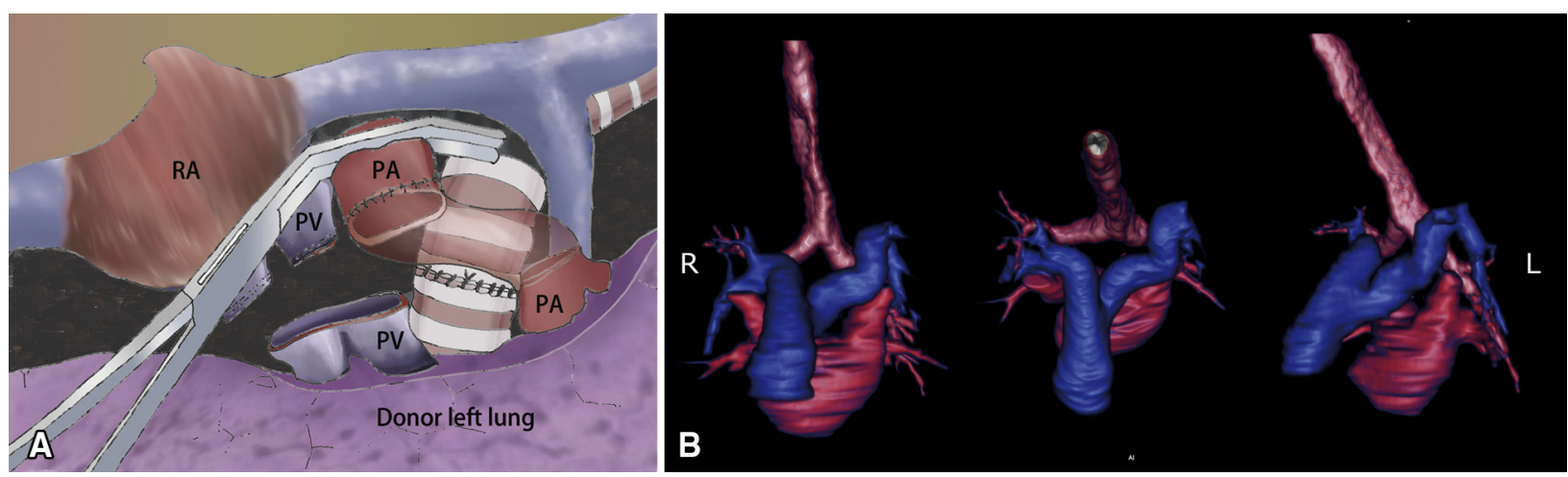

FIGURE 1. A, Graphic illustration of the surgical view during left lung implantation. Situs inversus will be present in patients with Kartagener syndrome. For left hilar anatomy, the left pulmonary artery $(P A)$ will be anterior to the airway and is usually referred to as the prebronchial PA. In situs solitus, the left PA will be superior to the airway (epibronchial PA). Adequate mobilization and preservation of the length of the donor PA are essential for vascular anastomosis. In both of our patients, we had to transpose the donor PA to the prebronchial location and end-to-end anastomosis was performed. B, Three-dimensional reconstruction of a patient with Kartagener syndrome after bilateral sequential lung transplantation (patient 2) illustrating the spatial relationship between the airway and vascular structures. The resultant left PA had a tortuous course from the prebronchial (anterior to bronchus) to epibronchial (superior to bronchus) location and the right PA had a configuration similar to the usual configuration found in situs solitus. Blue indicates the PA; red, the pulmonary vein $(P V)$ or left atrium; and white, the tracheobronchial airway. $R A$, Right atrium. 


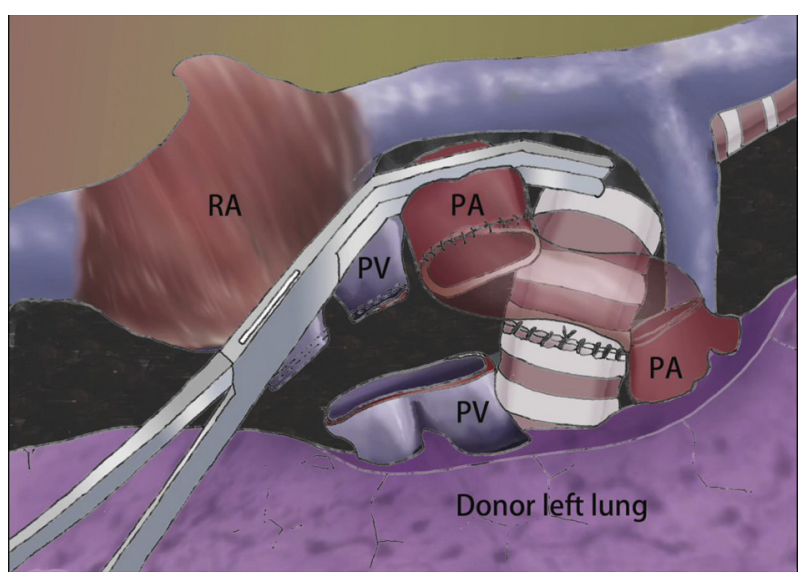

VIDEO 1. Video summary of the case showing the anatomy in Kartagener syndrome, the technique of hilar structure anastomosis during lung transplantation, and the result. Video available at: https://www.jtcvs.org/ article/S2666-2507(20)30303-5/fulltext.

epibronchial and prebronchial location. For airway anastomosis, 2 methods have been proposed. Some surgeons have reported the need to preserve a longer donor bronchus to mitigate the tension. ${ }^{3,4}$ Others have suggested that no alternations are needed, and the latter was the approach we adopted in both patients. ${ }^{1,5}$ A lung volume mismatch is more common on the right side because of dextrocardia. Some investigators have proposed that when the $\mathrm{D} / \mathrm{pR}$ index (donor/recipient predicted total lung capacity ratio) is $>1.12$, significant disparity will be present, and lung volume reduction will be necessary. ${ }^{5}$ However, we had had to perform lung volume reduction in patient 1 , although the $\mathrm{D} / \mathrm{pR}$ index had been $<1.12$. Although nonanatomic wedge resection should suffice usually, lobectomy could be required in extreme cases. A middle lobectomy should be preferred because the bronchial stump can be protected by the remaining lobes. ${ }^{3,6}$ Some surgeons have even opted for lobar lung transplantation instead. ${ }^{5}$ For lobar lung transplantation, it has been recommended to perform the lobectomy in the recipient lung and ensure lobar-to-lobar bronchial anastomosis to avoid stump complications. ${ }^{6}$ Intraoperative ECMO support has been shown to be associated with a lower incidence of primary graft dysfunction, bleeding, and renal and respiratory complications. ${ }^{7}$ However, it can limit cardiac drainage and heart manipulation owing to the preload-dependent nature of the centrifugal pump, lack of venous reservoir, and suboptimal flow control. ${ }^{8}$ Nevertheless, a stable hemodynamic state was achieved in both of our patients with ECMO support.

\section{CONCLUSIONS}

We have reported 2 cases of successful bilateral sequential LTx in patients with KS. LTx for such patients requires careful surgical planning and appropriate technical modifications with respect to the anatomic variations (Video 1).

\section{References}

1. Gauthier JM, Takahashi T, Bierhals AJ, Brody SL, Hachem RR, Witt CA, et al. Technical considerations for lung transplantation in Kartagener's syndrome. Ann Thorac Surg. 2019;107:e337-9.

2. Yazicioglu A, Alici IO, Karaoglanoglu N, Yekeler E. Pitfalls and challenges of lung transplant in a patient with Kartagener syndrome and scoliosis. Exp Clin Transplant. 2018;16:237-41.

3. Macchiarini P, Chapelier A, Vouché P, Cerrina J, Ladurie FL, Parquin F, et al. Double lung transplantation in situs inversus with Kartagener's syndrome. Paris-Sud University Lung Transplant Group. J Thorac Cardiovasc Surg. 1994;108:86-91.

4. Mentzer SJ, Aranki SF, Reilly FF, DeCamp MM, Hartigan P, O'Donnell W, et al. Single-lung transplantation in situs inversus. Ann Thorac Surg. 1994;58:1176-8.

5. Brioude G, D'journo XB, Reynaud-Gaubert M, Thomas PA. Bronchial fistula after lobar size reduction for bilateral lung transplantation in Kartagener's syndrome: a surgical challenge. Interact Cardiovasc Thorac Surg. 2013;17:184-6.

6. Kayawake H, Chen-Yoshikawa TF, Aoyama A, Motoyama H, Hamaji M, Hijiya K, et al. Surgical management of bronchial stumps in lobar lung transplantation. J Thorac Cardiovasc Surg. 2018;156:451-60.

7. Magouliotis DE, Tasiopoulou VS, Svokos AA, Svokos KA, Zacharoulis D. Extracorporeal membrane oxygenation versus cardiopulmonary bypass during lung transplantation: a meta-analysis. Gen Thorac Cardiovasc Surg. 2018;66:38-47.

8. Taka H, Miyoshi K, Kurosaki T, Douguchi T, Itoh H, Sugimoto S, et al. Lung transplantation via cardiopulmonary bypass: excellent survival outcomes from extended criteria donors. Gen Thorac Cardiovasc Surg. 2019;67:624-32. 